\title{
Load Modulation For Leadless Pacemaker Synchronization In A Dual Chamber Pacemaker System
}

\author{
Deepak Palaksha ${ }^{1}$ and Giulia Rizzo ${ }^{2,3}$, Ronald Nocua $^{2}$, Elie Lefeuvre ${ }^{3}$, Kimmo Kansanen ${ }^{1}$ \\ 1 - Department of Electronic Systems, NTNU, Norway \\ 2 - ValoTec, 1 Mail du Professeur Georges Mathé, 94800 Villejuif, France \\ 3 - C2N, Université Paris-Sud, Bât 220, rue André Ampère, 91405 Orsay cedex, France \\ Email: deepak.palaksha@ntnu.no giulia.rizzo@valotec.com
}

\begin{abstract}
Leadless Pacemakers are a new generation of pacemakers, providing therapy for patients with bradyarrhythmias. Studies are performed to extend the existing single chamber pacing to dual chamber pacing system. For such a system, the implanted leadless pacemakers should be synchronized to provide atrium and ventricular co-ordination.

Given size constraints from the first commercial products, the signal transmission efficiency has been investigated for varying distances between the two devices. The two devices are coupled thanks to electromagnetic inductive resonance. The energy consumed for providing synchronization is evaluated and is compared to available device battery capacity $(220 \mathrm{mAh})$, in order to test feasibility and estimate longevity. Device longevity plots for both simulation and experimental studies, for varying distances are analyzed, it is found that predicted longevity is less than 20 days (experimental study) for distances greater than $3 \mathrm{~mm}$.

In this paper, electromagnetic resonance coupled load modulation technique is considered for providing synchronization and is tested for feasibility in simulation and experimental studies. In general, the results indicate that the load modulation technique is feasible to provide synchronization in between leadless pacemakers for small distances $(<15 \mathrm{~mm})$ with a decrease in system longevity.
\end{abstract}

Keywords - Leadless pacemaker synchronization, inductive communication, load modulation in the leadless pacemaker, bio-circuits and system

\section{INTRODUCTION}

Pacemakers are implanted in patients with bradyarrhythmias. Innovation in pacemakers has led to many different configurations of pacing like single, double and multichamber transvenous systems. In the last years, pacemaker therapy has considerably expanded, exceeding 700000 implantations annually worldwide [1].

Despite the progress, pacemaker therapy still involve some complications, with possible dislodging of implanted lead from the pacemaker can [2]. In some cases, implantation also lead to complications like infection in valves. This issue has given way to develop a new system of pacing by eliminating leads; such devices are called leadless pacemakers.

The capsule has embedded electronics for pacing and sensing. A battery source powers the electronics, which is encapsulated in a biocompatible material. In the proposed leadless pacemaker unit there is overhead in circuitry which is used for synchronization of leadless pacemakers.

Traditionally single-chamber pacemakers, both atrial and ventricular devices, make up $<10 \%$ of pacemaker implants; whereas dual chamber pacemakers make up to $49 \%$ of pacemaker implants. Dual-chamber pacing allows for atrioventricular synchrony, which has been shown to minimize pacemaker syndrome [3]. The most significant limitation of the existing leadless pacemaker devices is the restriction to single-chamber, ventricular pacing. Extending the system from single chamber system to a dual chamber system can be achieved by establishing synchronization between leadless pacemakers, in other terms to maintain atrial-ventricular delay in the desirable range [5].

Electromagnetic inductive communication is a shortrange technique, based on the signal transmission between two resonant coupled circuits [4]. This configuration is mostly used in RFID devices, and it is innovative for the cardiac environment, especially for a leadless pacemaker. The two resonant coupled circuits are implemented in the leadless pacemaker implants at the right atrium and the right ventricle.

The synchronization between these leadless pacemaker implants is achieved by sending a carrier signal from the transmit unit (ventricle leadless pacemaker) to the receiver unit (atrial leadless pacemaker). The load at the receiver unit is modulated, based on atrial depolarization activity, at every beat. The variation of load in the receiver unit is reflected at the transmit unit as voltage deviation. The point in time where the deviation occurs is used as a time reference to establish the synchronization between leadless pacemakers. One of the advantages of using this strategy is that the receiver unit can be implemented with no overhead power consumption for synchronization.

In this paper, the device feasibility is tested, considering design constraints like coil size, and energy consumption at transmit resonance circuit. The paper provides analysis of 
load modulation technique to synchronize leadless pacemakers.

The paper is structured to give background in section II on dual chamber pacing and DDD pacing mode, and their design constraints. In section III, a system model for synchronizing leadless pacemakers using load modulation is provided. Section IV consists of simulation and experimental results, and feasibility analysis of the system. Section V contains conclusion and outline of our future work.

\section{BACKGROUND}

\section{A. Wireless DDD mode (RF-Pulse synchronization)}

If a dual chamber leadless pacemaker system operates in DDD pacing mode, such pacing mode is termed as wireless DDD mode [5]. The system has two leadless pacemakers, at the apex in the right ventricle (RV) and next to the sinoatrial (SA) node in the right atrium (RA). The two leadless pacemakers are independent pacing units, they measure the electrical activity at the placement site and pace locally as shown in Fig. 1.

The technology is different from the traditional dual chamber pacemaker system, as there is no pacemaker unit to synchronize the activity of implanted leadless pacemakers. In our previous work, an RF pulse was used to perform the synchronization between units, but, in this paper, the resonant coupling load modulation technique is tested for feasibility [5].

The DDD pacing mode provides coordination between the atrial and ventricular depolarization, by maintaining the following parameters:

1. Atrial-Ventricular delay (AV-delay): the mechanism to hold the electrical impulse at AV-node for a brief time (AV delay). This is to ensure that the atrium has completely ejected blood into the ventricle. In nutshell, this is the delay in time between atrial and ventricular contraction.

2. Offset time $\left(\mathrm{T}_{\text {offset }}\right)$ : time delay in electrical conduction pathway from the atrium to ventricle, due to pacing at the atrium. This indicates the extra time needed for the electrical impulse to reach the atrium when compared to natural electrical conduction.

In our previous work, these parameters were communicated from atrium to ventricle by employing an RF pulse transmission and synchronized by an algorithm [5].

\section{B. Electromagnetic resonance coupled load modulation}

There are several techniques that use electromagnetic communication. In this case, the resonant inductive communication is chosen for implanted devices which are separated by small distances. This technique is commonly employed to wirelessly charge implantable devices; in fact, it allows to transport $\mathrm{mW}$-power without too many losses through the human body. The electromagnetic transmission is also involved in data communication, thanks to inductive resonant circuits. Passive radio frequency identification systems (RFID) use this technology extensively, but it is a

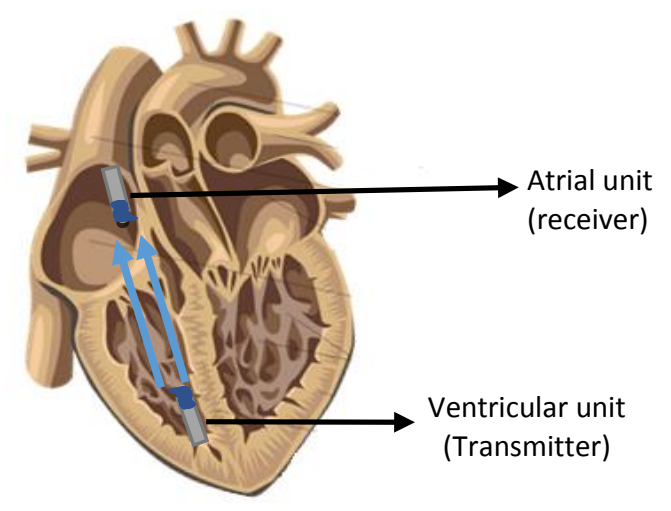

Fig. 1: Dual chamber leadless pacemaker system, the coils are represented by blue region in both leadless pacemakers.

novel strategy for cardiac environment [4].

The schematic representation of Fig.2 represents the electronic system components. It consists of two main units:

- Transmit block

- $\quad$ Receiver block

Each part is formed by two coils and a resonant circuit. The transmit part consists of the power supply, the resonant circuit, containing the transmitter coil (Tx), and the envelope detector circuit, to detect the voltage deviation. The receiver block is constituted by the receiver coil $(\mathrm{Rx})$, the resonant circuit, the load, and a switch to modify the load.

The carrier wave is transmitted from the transmitter coil to the receiver one, and, in response, the impedance of load at receiver block is modulated, depending on the atrial contraction. This modulation is reflected on the transmit carrier signal as a voltage deviation across the transmitter coil. A demodulation circuit at the transmitter detects the voltage deviation. The application of this technique is used to provide coordination between the atrium and ventricle while implementing a wireless DDD mode.

\section{Design specification}

Medical implants have different constraints like material used, energy consumption, and size. The involved materials

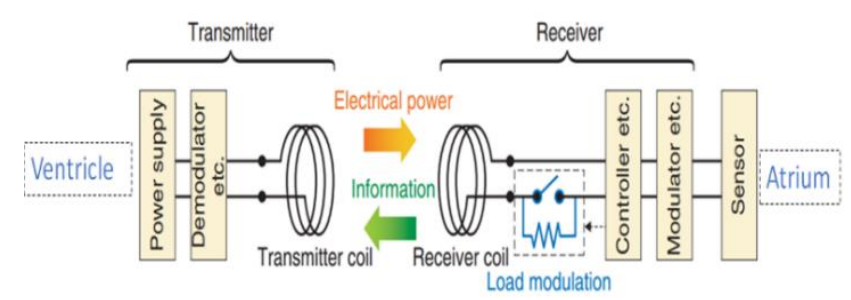

Fig. 2: Electronic scheme of load modulation circuits ${ }^{[4]}$ 
must guarantee the biocompatibility and the hermeticity of the device. From the point of view of energy consumption, the device must satisfy the system functionalities and at the same time, it must conduct it in the battery's lifetime. Furthermore, the size constraints are mostly dependent on the battery dimensions.

In the case of leadless pacemakers, the existing device has a length and a thickness, in average, of $42 \times 6.7 \mathrm{~mm}$ [6]. $30 \mathrm{~mm}$ of them are used for the inherent components, which handles the pacemaker functionalities, the other $10 \mathrm{~mm}$ are used for the inductive coils. Hence in this study, the transmitter and receiver coil are made of $10 \times 6.5 \mathrm{~mm}$ dimension [6]. Furthermore, energy feasibility analysis considers an available battery capacity of 220mAh [7], the energy consumption for synchronization is compared to the available battery energy to predict system feasibility.

\section{SYSTEM DESCRIPTION}

In this section, dual chamber leadless pacemaker system, pacing in wireless DDD and synchronizing through load modulation, is detailed. In addition, synchronization methodology, employed to overcome the shortcomings of traditional single chamber leadless pacemaker, is described in the following paragraphs.

\section{A. Wireless DDD mode (load modulation synchronization)}

The proposed leadless pacemakers are implanted in the atrium and ventricle, as shown in Fig. 1. For implementing a wireless DDD pacing mode, the ventricle unit needs to know the expected time of ventricular depolarization $\left(\mathrm{T}_{\mathrm{vpred}}\right)$. The load modulation provides, in this case, the time reference to calculate $\mathrm{T}_{\text {vpred. }}$.

At the ventricular unit, the size of the observation window is obtained from atrial window size $\left(\mathrm{T}_{\text {asmin }}, \mathrm{T}_{\text {asmax }}\right)$. This ensures that the atrial activity is not missed in the observation window. The simplified functional block diagram for leadless pacemakers is shown in Fig. 3.

The sequential steps performed for providing synchronization is ( Fig. 4):

- The atrium and ventricle are sensed for depolarization activity and the sensing window ranges are $\left[\mathrm{T}_{\mathrm{asmin}}, \mathrm{T}_{\text {asmax }}\right]$ and $\left[\mathrm{T}_{\mathrm{vsmin}}, \mathrm{T}_{\mathrm{vsmax}}\right]$ respectively.

- A carrier signal is transmitted from transmit circuitry (ventricle unit) during the observation window $\left[\mathrm{T}_{\mathrm{rxmin}}\right.$, $\left.\mathrm{T}_{\text {rxmax }}\right]$.

- At atrial unit, a load $\left(\mathrm{R}_{\mathrm{L}}\right)$ is connected in parallel to the receiver resonant circuit based on, if it is natural contraction or paced case.

- Load is connected instantly if the atrium depolarizes due to natural contraction, and after time delay ( $\mathrm{T}_{\text {offset }}$ ), if atrium is paced.
- The resulting voltage deviation is detected at transmit coil (ventricle unit) by envelope detector circuit and corresponding time is $\mathrm{T}_{\mathrm{Rx}}$.

- After obtaining $T_{\mathrm{Rx}}$, the ventricle unit expects the spontaneous ventricular depolarization to occur at $\mathrm{T}_{\mathrm{vpred}}=$ $\mathrm{T}_{\mathrm{Rx}}+\mathrm{T}_{\mathrm{avd}}$, where $\mathrm{T}_{\mathrm{avd}}$ is the pre-programmed AV delay

(see Fig. 4)

- If no ventricular depolarization was sensed within $\left[\mathrm{T}_{\mathrm{vsmin}}\right.$ $\left.\mathrm{T}_{\mathrm{vsmax}}\right]$, then the ventricular unit will pace the ventricle.

The operation is repeated on every beat, achieving atrialventricular coordination. Resonance coupled load modulation is discussed in the next subsection.

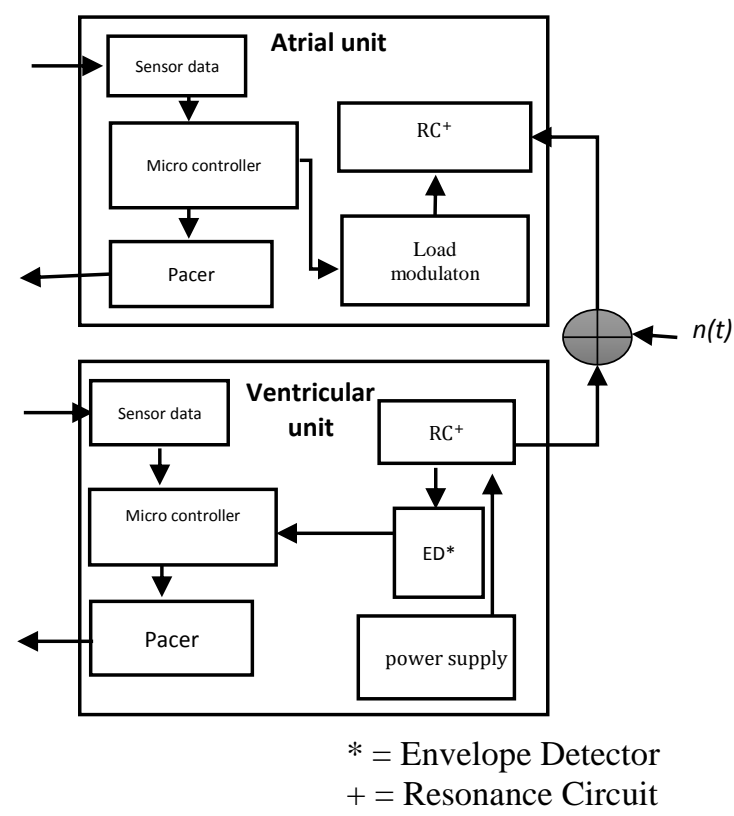

Fig. 3 Functional block diagram

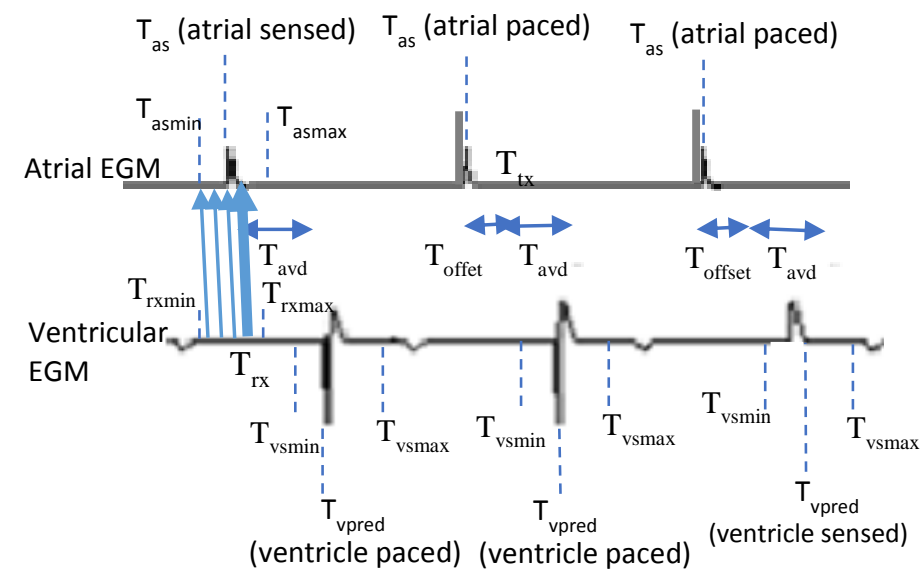

Fig. 4 Timing diagram of synchronization mechanism, the change in the width of arrow head in the observation window $\left[\mathrm{T}_{\mathrm{rxmin}}, \mathrm{T}_{\mathrm{rxmax}}\right]$ represents a change in load at atrial unit due to atrial depolarization. 


\section{B. Synchronization methodology and study setup}

Synchronization circuit consists of a carrier signal generator and LC circuit. The setup is studied in order to have the highest transmission efficiency, which is directly dependent on two parameters, the coupling coefficient and the quality factor, respectively Eq.(3) and Eq.(4). The coupling coefficient $(\mathrm{k})$ provides the degree of electromagnetic coupling between resonant circuits, it is a function of distance and coil specification [8]. The quality factor (Q) gives information about the coil's electromagnetic performance, it depends on the frequency and on the inductor's internal resistance [8].

As already explained in Paragraph II.C, the size constraints for the leadless pacemaker are linked to a maximum length of $10 \mathrm{~mm}$, a diameter of $6.5 \mathrm{~mm}$, the cylindrical shape, and the operating frequency adopted is 13.56MHz. In fact, a carrier frequency of $13.56 \mathrm{MHz}$ is a general value, chosen on the base of the frequency band assignments for medical devices and tissue absorption criteria [9].

For all these reasons, the cylindrical coils are made of dimensions, coil length $=(10 \pm 1) \mathrm{mm}$ and diameter $=(6.5 \pm 1)$ $\mathrm{mm}$. The material used to design the coil is Litz wire which allows having fewer losses. It has a wire diameter of $0.75 \mathrm{~mm}$, and the turns are fixed with fevikwick glue. The highest number of turns achieved for this coil configuration is 10 turns. It is possible to theoretically calculate its inductance with the formula:

$$
L=\frac{\mu_{0} N^{2} A}{l}
$$

The transmitter and receiver coils have a theoretical inductance of $0.42 \mu \mathrm{H}$ and both of them are symmetric. Measuring the inductance with the impedance analyzer, Hewlett Packard 4194A Impedance Analyzer, the best value in terms of quality factor, results in inductance $=0.4 \mu \mathrm{H}$ at the frequency of $16 \mathrm{MHz}$. This deviation from the theoretical expectation is due to the handmade coils.

Then it is possible to estimate the values of the capacitors, necessary for the resonant circuit at the experimental frequency of $16 \mathrm{MHz}$. From the theoretical point of view, the value of the capacitor is $0.25 \mathrm{nF}$, and it can be calculated with the Eq.(2):

$$
C=\frac{1}{\left(2 \pi f_{r}\right)^{2} L}
$$

From these data, the system's efficiency can be estimated, starting from the coupling coefficient (k). In the case of cylindrical coils, it is given by Eq.(3):

$$
k=\frac{d^{3}}{\left(d^{2}+D^{2}\right)^{\frac{3}{2}}}
$$

where $d$ is the coil diameter and $D$ is the distance between coils [8].

The Q factors of the transmit and receiver coil are around 6.7 measured from the impedance analyzer, and it is not too high, and theoretically, they are calculated from Eq.(4). It

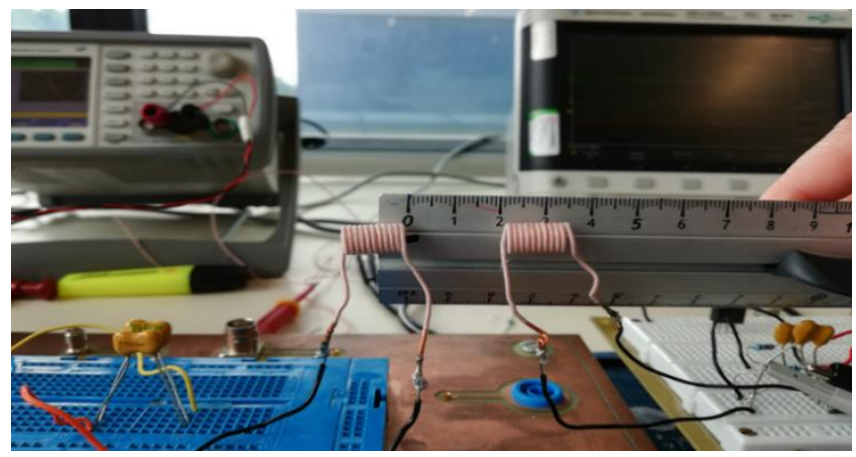

Fig. 7 Experimental setup for a distance of $20 \mathrm{~mm}$ between transmitter and receiver coils

has a theoretical $\mathrm{Q}$ factor $=34.27$ and the difference in the values is due to handmade coils.

$$
Q=\frac{2 \pi f L}{R}
$$

where $f$ is the carrier frequency, $L$ is the inductance and $R$ is the coil's internal resistance which is $1 \Omega$ [9]. The power transfer efficiency of the transmitter coil is given by Eq.(5):

$$
\eta=\frac{k^{2} Q_{1} Q_{2 L}}{1+k^{2} Q_{1} Q_{2 L}} \frac{Q_{2 L}}{Q_{L}}
$$

Where $Q_{1}$ and $Q_{2}$ are Q-factor of Tx and Rx coils, $Q_{2 L}=\frac{Q_{2} Q_{L}}{\left(Q_{2}+Q_{L}\right)}$ and $Q_{L}=\frac{R_{L}}{2 \pi f L_{2}}, R_{L}$ is the load resistor at the receiver circuit [9].

In order to not only transmit the carrier signal but also to detect the modulation of the voltage (voltage deviation) in the transmitter block, an envelope detector circuit is inserted across coil for detecting the voltage deviation. The point in time where deviation is detected $\left(T_{t x}\right)$ is used as a reference time to calculate the ventricular depolarization time $\left(\mathrm{T}_{\mathrm{vpred}}\right)$. Most of the existing RFID techniques use the envelope detector circuit for demodulation because of its low complexity and low power consumption characteristics, therefore envelope detector is considered for analysis. The envelope detector circuit has a detectable voltage threshold $\approx 200 \mathrm{mV}$ [11], so the transmit resonant circuit is tested for deviation of at least $200 \mathrm{mV}$, due to change of load in the

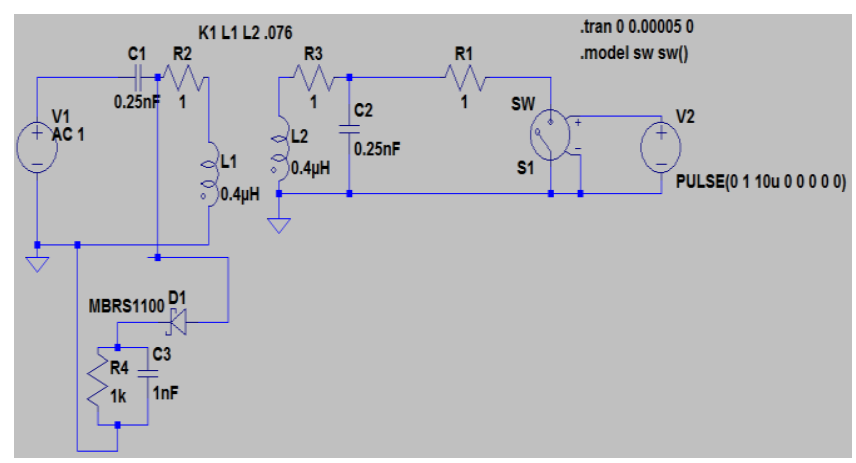

Fig. 6 Circuit simulation in LT Spice 
receiver block. The values for the resistor and capacitor of envelope detector are chosen such that the time constant is much less than the carrier frequency $\left(f_{r}\right)$ [10]. Currently used resistance and capacitance values are $1 \mathrm{k} \Omega$ and $1 \mathrm{nF}$, as shown in Fig. 6. There are alternative approaches to design envelope detector circuit as done in [12], but envelope detector design is not the major focus of this paper.

Regarding the receiver block, the resonant circuit is connected in parallel to a load resistor with a switch. When atrium is depolarized it is indicated at atrial unit by connecting the load to the resonant circuit. The change in load, in the receiver (atrial unit) block, is reflected at the transmitter coil (ventricle unit) and detected by the envelope detector circuit. The value of the load resistance is $1 \Omega$ (see Fig. 6) and the voltage deviation at the output of the envelope detector increases with the decrease in load resistance. The schematic for the transmitter and the receiver circuit is shown in Fig. 6. LT spice is used for analysis as a simulation tool. Fig. 7 illustrates the experimental setup for the study. The performed analyses have three main objectives:

- Measuring power transfer efficiency between the transmitter and receiver resonant circuits, varying the distance of the two blocks.

- Evaluation of the required transmitter voltage, to maintain the detection threshold of $200 \mathrm{mV}$ at different distances.

- Estimation of the system longevity for varying distance between transmit and receive coils.

\section{Power consideration}

The major focus of the paper is to analyze system feasibility and its longevity. Leadless pacemaker consumes around $8-10 \mu \mathrm{W}$ for all electronics, including the pacing and communication transceiver, and the expected longevity time is 10 years [6]. In this paper, the feasibility analysis is performed by comparing the energy consumed by synchronization strategy to available battery energy. The consumed energy per beat, for synchronization, is the product of instantaneous power and the observation window $\left(100 \mathrm{~ms}\right.$ ). Instantaneous power is calculated as $\frac{V 1^{2}}{R_{2}}$ (see Fig. 6). Furthermore, the longevity of the system is calculated as a function of the distance, considering a constant pacing rate of $60 \mathrm{bpm}$. $60 \mathrm{bpm}$ implies the synchronization of atrium and ventricle is performed every $1 \mathrm{sec}$. Therefore, longevity is evaluated by calculating time needed by energy per beat to drain the available battery (220mAh).

\section{RESULTS AND DISCUSSION}

In the current study, the load modulation feasibility is tested in terms of energy consumption and longevity. The power transfer efficiency, between the transmitter and receiver blocks, directly depends on the coupling between the two coils and on the quality of their resonant circuits. As shown in Fig. 8, the received power at the atrial unit

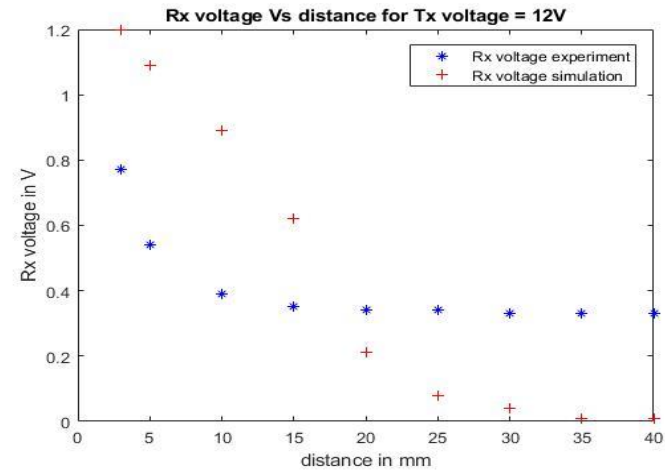

Fig. 8 Received voltage on $\mathrm{Rx}$ vs distance between transmitter and receiver coil, for a transmit voltage of $12 \mathrm{~V}$.

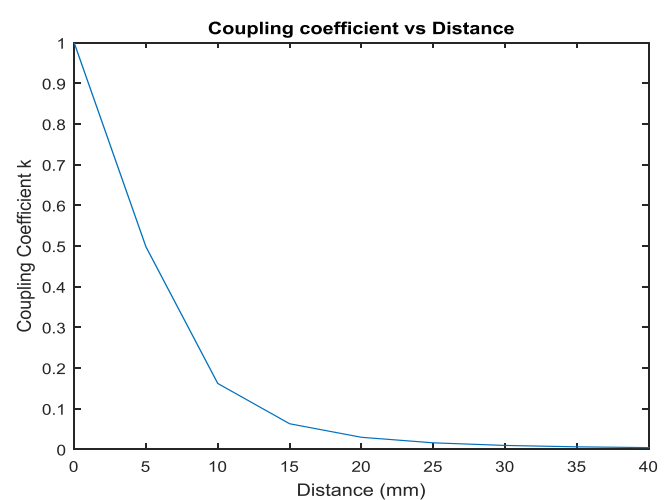

Fig. 9 Trend of Coupling coefficient at different distances

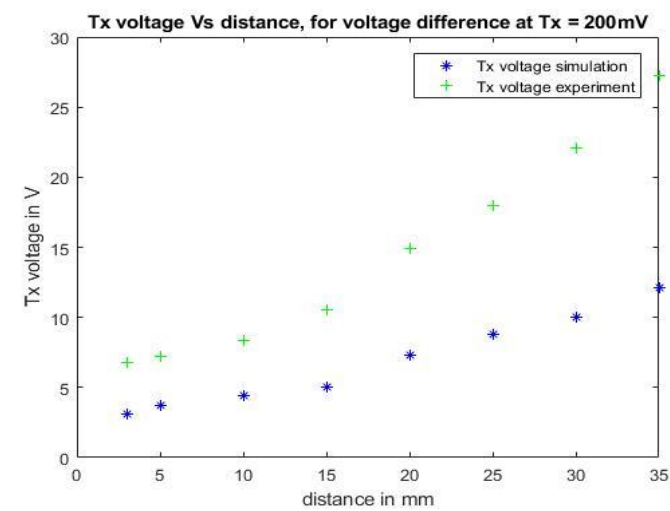

Fig. 10 Transmitted voltage vs distance between transmitter and receiver coil, for a detectable threshold voltage of $200 \mathrm{mV}$.

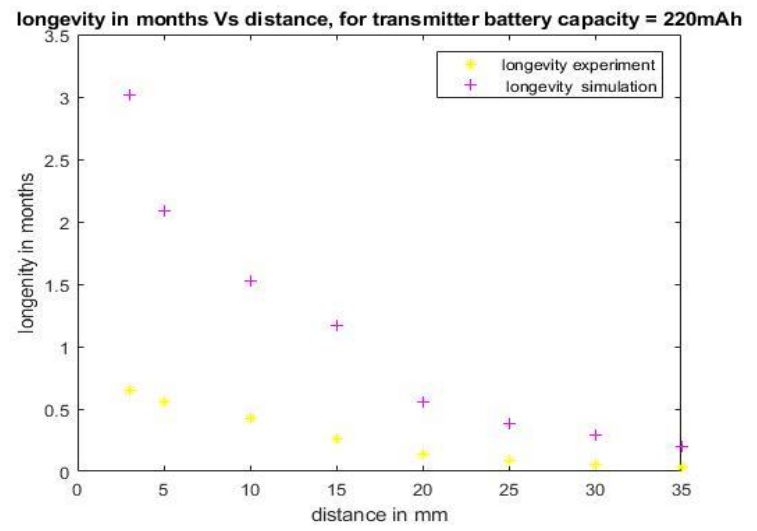

Fig. 11 Longevity estimation [months] vs distance between Tx and Rx coils, with a battery capacity of $220 \mathrm{mAh}$. 
decreases when the distance between $\mathrm{Tx}$ and $\mathrm{Rx}$ coils increases. For a transmit voltage of $12 \mathrm{~V}$, the received voltage drops around $0.2 \mathrm{~V} / 5 \mathrm{~mm}$ until $15 \mathrm{~mm}$, and drops rapidly after $15 \mathrm{~mm}$.

This is coherent with the theoretical model, in fact, when the two coils are further, the captured electromagnetic-field lines are less than the ones at $0 \mathrm{~mm}$-distance. The values of power are measured at a fixed applied voltage across transmit coil, $12 \mathrm{~V}$. Furthermore, after $15 \mathrm{~mm}$ the received power in experimental results saturate due to channel noise. The power transfer efficiency of the resonant circuit was calculated from the equation (5) and the trend followed Fig. 8. It is possible to see this behavior in the theoretical graph of the coupling coefficient $(\mathrm{k})$, as a function of the distance between coils, Fig.9.

Fig. 10 illustrates the applied voltage, necessary to obtain a successful detection over varying distance. The results show that there is a significant increment of the supplied power, in order to achieve the detectable threshold voltage of $200 \mathrm{mV}$ in the ventricular unit. This trend shows a deviation from the simulation model, due to losses in the coil during measurements, more relevant at higher distances. The plot also shows the limitation of the system in case of longer distance between the transmitter and receiver circuits.

The following plot (see Fig. 11) shows the expected longevity of the device at different distances, estimated for a battery of $220 \mathrm{mAh}$. Longevity is evaluated as described in section III.C. It is seen that both simulation and experimental analysis follows the same trend but the longevity in case of experimental analysis is less than simulation, this is due to losses in coil in practical setup. It is clear that the system longevity is tremendously low when compared to the average life cycle of pacemakers (10 years). For a distance separation of $3 \mathrm{~mm}$ between transmitter and receiver block, the longevity is estimated to be around 3 months and 20 days in simulation and experimental analysis respectively.

Furthermore, all the analyses do not consider the attenuation due to the casing around the coil, which would further decrease the received power and have a feedback on the measured modulation. It will be investigated in our future work. Also, other strategies are studied to implement adaptive transmission to conserve energy.

\section{CONCLUSION}

In the dual chamber leadless pacemaker system, Wireless-DDD pacing mode is tested. The system feasibility is investigated, by establishing synchronization between leadless pacemakers using a resonance load modulation technique.

Coils are designed, starting from the size constraints of the leadless pacemaker, already available in the market. They are designed symmetrically, coaxially, with the same dimension, using litz wire. The use of litz wire resulted not a good option for small coils as in this case. The transmission of signal could be incremented using copper wire.

At the atrial unit, the load is modulated by connecting a load resistor $\left(R_{L}\right)$ in parallel to the resonant circuit, to indicate the atrial depolarization. Furthermore, an envelope circuit in ventricle unit detects the voltage deviation and, from this reference in time, it predicts the ventricular depolarization time $\left(\mathrm{T}_{\mathrm{vpred}}\right)$ to provide synchronization.

For a given transmitted power, the received power is evaluated for varying distances. The power transfer efficiency of resonant circuits is analyzed. The necessary transmitted power, to achieve the detection threshold ( $200 \mathrm{mV}$, value chosen to overpass the backgroung noise), is evaluated in simulation and in experimental studies. It is seen that the required transmitted voltage increases rapidly, when the two coils are further than $15 \mathrm{~mm}$ (Fig. 10). So, the feasibility of the system, for this setup, seems to be efficient until $15 \mathrm{~mm}$ of distance.

Longevity of the system is calculated by extending the evaluation of energy consumed per beat. The predicted longevity in simulation and experimental setup is provided for different distances (Fig. 11). The results demonstrate that the strategy is not promising with considered system condition and design.

The system has to be optimised from the point of view of the coils, from the materials to geometry, and the system, increasing the total quality factor. The load modulation is still a new technique, not explored in leadless pacing and sensing. As said before, to be feasible and competitive with the new generation batteries, employed in the cardiac devices, the architecture has to be optimsed in the future works.

\section{ACKNOWLEDGMENT}

This work is funded by the European Union's H2020: MSCA: ITN program for the "Wireless In-body Environment Communication - WiBEC" project under the grant agreement no. 675353 . We would like to thank people from Valotec for extending their support for the research.

\section{REFERENCES}

1. Mond HG, Proclemer A. The 11th world survey of cardiac pacing and implantable cardioverter-defibrillators: calendar year 2009da World Society of Arrhythmia's project. Pacing Clin Electrophysiol. 2011;348:1013e1027.J.

2. Kirkfeldt RE, Johansen JB, Nohr EA, et al. Complications after cardiac implantable electronic device implantations: an analysis of a complete, nationwide cohort in Denmark. Eur Heart J. 2014;35:1186e1194.

3. Mohd Noor IslamaMehmet R.Yuceb . Review of Medical Implant Communication System (MICS) band and network, ICT Express, Volume 2, Issue 4, 2016, Pages 188-194,ISSN 2405-9595.

4. Akimoto, M \& Iizuka, M. (2013). Load modulation applied to magnetic resonance wireless power transfer technology and its applications. 
5. Palaksha et al., Feasibility Analysis for Pulse Based Synchronization In A Dual Chamber Leadless Pacemaker System, Body Nets, Oct. 2018.

6. Skevos Sideris, Stefanos Archontakis, Polychronis Dilaveris, Konstantinos A. Gatzoulis, Konstantinos Trachanas, Ilias Sotiropoulos, Petros Arsenos, Dimitrios Tousoulis, Ioannis Kallikazaros, Leadless Cardiac Pacemakers: Current status of a modern approach in pacing, Hellenic Journal of Cardiology, Volume 58, Issue 6, 2017, Pages 403-410, ISSN 1109-9666.

7. Marc A. Miller, Petr Neuzil, Srinivas R. Dukkipati, Vivek Y. Reddy, Leadless Cardiac Pacemakers: Back to the Future, Journal of the American College of Cardiology, Volume 66, Issue 10, 2015, Pages 1179-1189, ISSN 0735-1097,.

8. Grandolfo, M., Vecchia, P. \& Gandhi, O. P." Magnetic Resonance Imaging: Calculation of Rates of Energy Absorption by a Human-Torso Model". Bioelectromagnetics 11, 117-128 (1990).

9. Abiri P., Moloudi S. \& Hsiai T. K., "Inductively powered wireless pacing via a miniature pacemaker and remote stimulation control system", 10.1038/s41598-017-06493-5

10. Current Wikipedia, The Free Encyclopedia, "Envelope detector"'[Online].Available:https://en.wikipedia.org/wiki/Envel ope_detector [Accessed: February 15, 2018].

11. P. N. A. Fahsyar and N. Soin, "CMOS implementation of envelope detector circuit in $0.18 \mu \mathrm{m}$ Process," 2010 IEEE International Conference on Semiconductor Electronics (ICSE2010), Melaka, 2010, pp. 145-148.

12. Puteri Nor Aznie Fahsyar SM and N. Soin, "Performance comparison of envelope detector for RFID applications," 2009 International Conference for Technical Postgraduates (TECHPOS), Kuala Lumpur, 2009, pp. 1-3. 